Témoigner Témoigner. Entre histoire et mémoire

Getuigen Revue pluridisciplinaire de la Fondation Auschwitz

$123 \mid 2016$

Traduire le témoignage

\title{
Des perspectives de conservation de quelques sites mémoriels locaux
}

Daniel Weyssow

\section{(2) OpenEdition}

1 Journals

Édition électronique

URL : https://journals.openedition.org/temoigner/5572

DOI : $10.4000 /$ temoigner.5572

ISSN : 2506-6390

Éditeur :

Éditions du Centre d'études et de documentation Mémoire d'Auschwitz, Éditions Kimé

Édition imprimée

Date de publication : 1 octobre 2016

Pagination : 216-220

ISBN : 987 2-9600926-4-6

ISSN : 2031-4183

Référence électronique

Daniel Weyssow, «Des perspectives de conservation de quelques sites mémoriels locaux »,

Témoigner. Entre histoire et mémoire [En ligne], 123 | 2016, mis en ligne le 02 novembre 2021, consulté le 04 novembre 2021. URL : http://journals.openedition.org/temoigner/5572 ; DOI : https://doi.org/ 10.4000/temoigner.5572

Tous droits réservés 


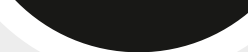

$\diamond$ Cette rubrique se propose d'éclaire les processus parfois construction mémorielle tels qu'ils se produisent dans différentes parties $d$ monde. Au lieu de se concentrer sur les événements, le Laboratoire mémoriel se penche sur les modalités de la mémoire collective, en mes défis les enjeux et les conséquences socio-politiques du travail de mémoire.

\section{DES PERSPECTIVES DE CONSERVATION DE QUELQUES SITES MÉMORIELS LOCAUX}

Sous quelles formes la mémoire de la Seconde Guerre mondiale est-elle montrée, rappelée, honorée et conservée dans notre espace public?

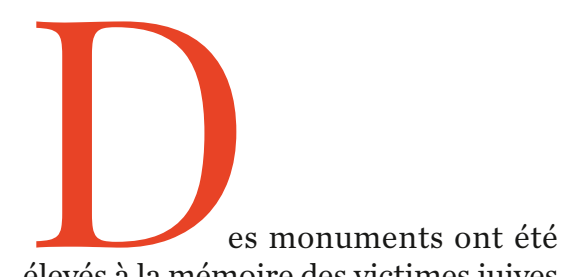
élevés à la mémoire des victimes juives et/ou résistantes, des bâtiments aménagés en Centres de documentation et en musées, et des plaques mémorielles apposées sur des immeubles emblématiques. Des artères ont été (re) baptisée et dernier avatar en date des paves et, dernier avatar en date, des pavés de ménoiresont domiciles des person aux derniers domiciles des personnes

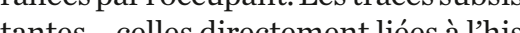
toire-les plus à même de recs âlhisde la mémoire des faits et surcopte de la ménoiro des faits et susceptibles den permettre la transmission peuvent encisureliste placesur une histe deprotectiondupatriments ris ils le souvenir L'oubli - l'effacement de la quili convient avant tout de neutraliser Lobstacle de la reconnaissance franchi, dans l', traces et temoignages inscrits dans lespace public seront-ils pou pour tou jamais conservés ? Comme pour tout ce qui existe, leur disparition, termit lait partie de lordre des choses En fait, la luttecontreles pertes d inforurbains d'oménagentions et les projets

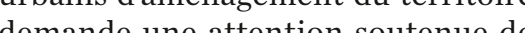
la part de ceux qui solhitent que le pácialistes, les associations dédiées, devecialstes, les associations dédieses, ne dénaient cesser, pourle sauvegarder, de et politiques, les « présens es du pres.

Divers exemples pris dans notre proche environnement évoquant la
Seconde Guerre mondiale peuvent nous faire prendre conscience de la fragilité de la mémoire dans l'espace public. Parmi les bâtiments et immeubles dotés dune connotation mémorielle de premier plan,le Fort de Breendonk. Celui-c le graurd public et les écoliers afin que chacun puise se rendre com que ćvices qui ont été portés a l'hpte des durant la Seconde Guerre durs son Seconde Guerre mondiale, tion son écélnte. Risquent la disparide l'humidité régnante dans le fort reffitis tracés par les intén lort, les graffits tracés par les internés sur les murs des cellules. Ces témoignages ont graphiés dès après la guerre

La Caserne Dossin ${ }^{2}$ camp de transit SS ayant servi de plaque tournante pour été transformée en Jur co Belgique, a été transformée en un complexe d'appartements. Laathenticité des lieux, si 'on tientà sen récerere crucial épisode esous-soldu porche d'entrée, elle n'est la part de ceux qui souhaitent que le SS ayant servide plaquetournante pour evoqué, à de la sorte été perturbée.

Paigh REFU Whe in $B$. Wer Go GIES

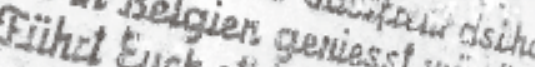
Quf. Achlot dis in nis stortionitig

Euch nicht dio Sitten wes fartiger 7 dor sicht bemechbar vernuidet

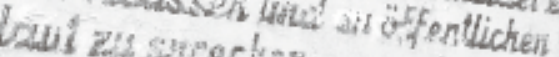

Wahset hivist.

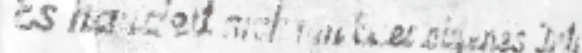

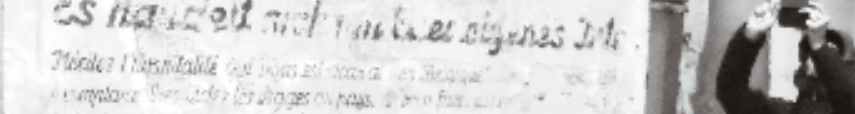
au public. Le musée Kazerne Dossin, qui aurait logquement dâ yetre amènagé, la finalement été, mais en y ent dé y sont idéalementreunies, là n'est pas la question, mais peut plu être ap

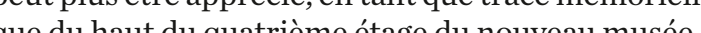
que du haut du quatrième étage du nouveau musée.

Au 25-27 de la rue van der Weyden à Bruxelles, non loin de la gare du Midi, se trouvait, juste avant-guerre, e Comité d'Assistance aux Réfugiés juifs ${ }^{3}$. Un mur de la cour intérieure conserve un texte peint, rédigé en demand et en français, suggérant des recommandations comportementales aux réfugiés afin qu'ils puissent se fondre discrètement parmi la population du quartier Ces inscriptions nurales ont récenment pers une évolution des dégradations. Le plâtre s'effrite en emportant les «écritures». Le propriétaire s'est-il manifesté auprès des Monuments et Sites? Comment pourrait-il être maintenu en l'état? Ces questions demandent de réponses urgentes si l'on souhaite que ce témoignage tout de même extraordinaire subsiste. (1) Olivier Van der Wilt, «Les détenus de la SIPO-SD en Belgique : des
caves de lavenue Louise aux cellulus de Breendonk», in Les Caves dela
Gestapo. Reconnaissance et conservation, Daniel Weyssow (dir.), Paris, Kimé, «Entre Histoire et Mémoire», 2013, p. 121-133,

(2) Philippe Mesnard, «Caserne Dossin (Malines). Le parti pris de la clarté et des droits de l'homme, in Témoignere. Entre histoire et mémoire. (3) Photographie du mur de la cour : http://marolles-memories.net/ff/ 


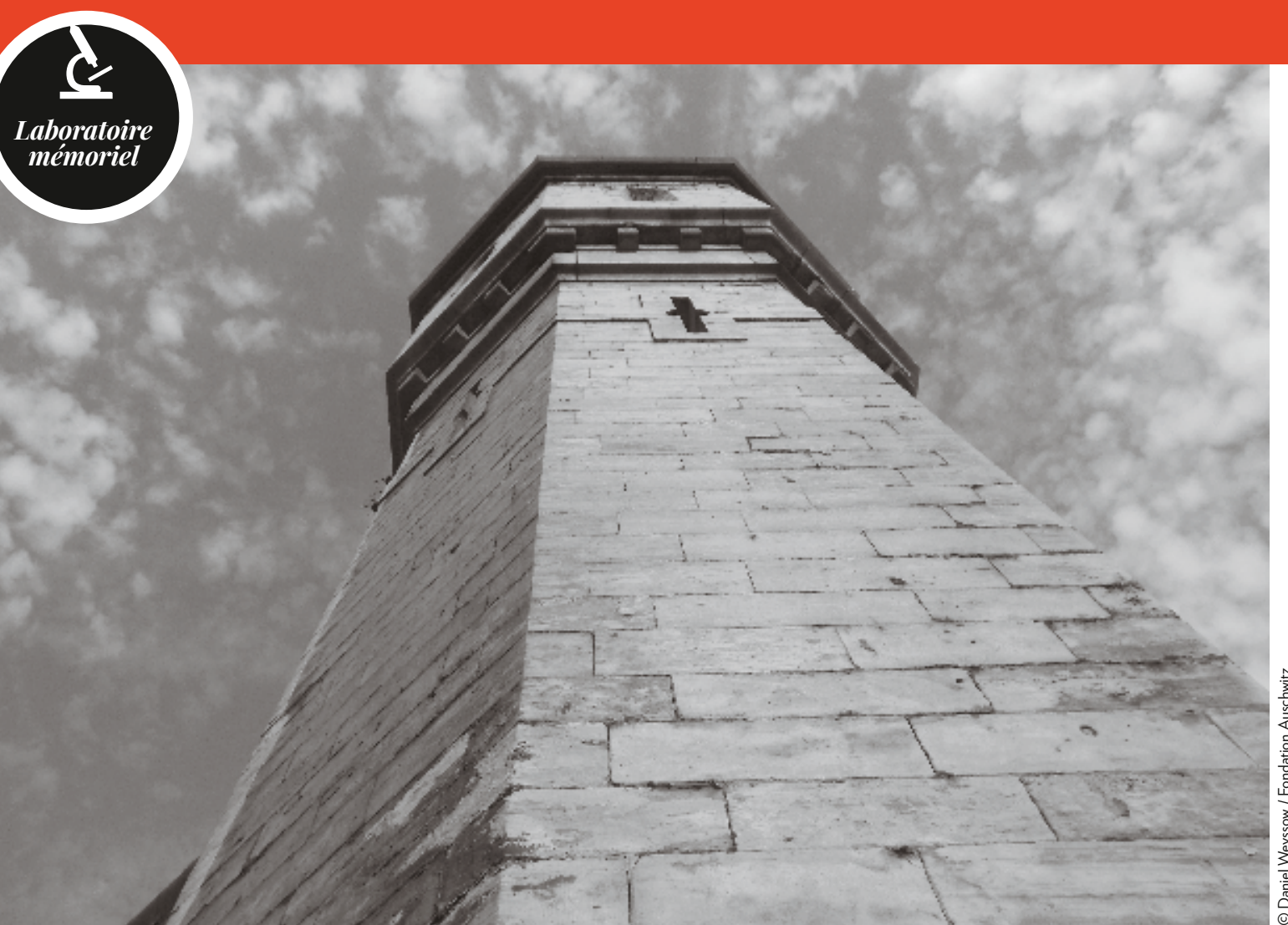

_La Prison de Saint-Gilles.

- Les traces gravées dans les caves du siège de la Gestapo ${ }^{4}$, aux 347 et 453 avenue Louise, étaient égalemen en bonne voie d'oubli. Si l'historien et cinéaste André Dartevelle navait pas, en 1995, cherche à explore d'un docin in dun doc'n nés par les victimes juives et résistantes qui y furent nés par les victimes juives et résistantes qui y furen séquestrees ont par miracle pourrait-on dire echappe quille promé Pourront-elles, puisqu'ellent tranquille programmé. Pourront-elles, puisquelles sont en vórie de classement definitie faire lobjet dune étude sérieuse et complète, les lieux restants à l'usage exclusi
des propriétaires?

La Prison de Saint-Gilles ${ }^{5}$ a compté en ses murs de très nombreux résistants arrêtés par l’occupant durant les deux guerres mondiales. À l'issue de la Première, la cellule d'Édith Cavell ${ }^{6}$ devint un mémorial. Elle était visitee chaque annee par une délegation anglaise qui
venait y honorer sa mémoire et sa bravoure, mais le

écent rafraîchissement de la prison semble bien avoi u raison de cette tradition. Ce n'était sans doute là qu'un avant-gôt de ce qui cepectait sens coute là fait, aux dernières nouvelles, que toute la prison aujourd'hu, à l'hon 2017 , menace par de démolition 7 . Il est en effet projeté d'y con projet un nouveau quartier d'immeubles doté d'enviro un millier d'appartements ${ }^{8}$ et d'une école. L'id́e de classer une partie du site fait partie du pu.t, mas ne s'ait pas d'en conserver l'ensemble La techis

(4) Les Caves de la Gestapo. Reconnaissance et conservation, Danie
Weyssow (dir.), op. cit.

(5) Photographie de la prison vue du ciel
570.buildings.10601043_0106_W04.JPG

photos/CAVELLEEdith_23038_17.jpg

(7) http://petitionspatrimoine.blogspot.be/

(8) http: ///www.ms-a.be/pdf/MSA\%20-\%20booklet\%202014\%20
URBANISME.pdf projetée semble même fort proche de celle qui fut adoptée pour la transformation de la caserne Dossin. Des témoignages poignants, prononcés par les vicde la Geheime Feldpolizei (GFP), font poustant prendre qu'il s'agissait d'un lieu maje dur conprelicier de loc supant Encore uje du dispositif policierdeloccupant. Encore une lois, revons le fait que Bruxelles ne conpte pas de lieu à la fois «istodedoum du

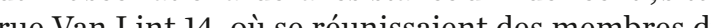
Front de l'Indépendance.
La caserne du Tir national ${ }^{10}$ et son terrain, incluan 'Enclos des fusillés ont été vendus en 1962 àl'Institut Nation de fiodifterión (INR) afn de laisser vaxima de

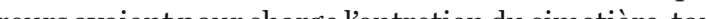
joursexistant

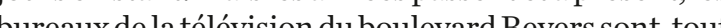
comme la Prison de Saint-Gilles, voús à une dispart-

(9) http://marolles-memories.net/fr/la-plaque-photogravee-du-faux-soi// (10) http:///www.bel-memorialororg/cities/bruxelles-brussel/schaerbeek/
tir_national/schaerbeek_tir_national_enclos_des_fusilles_cpa_07.jpg

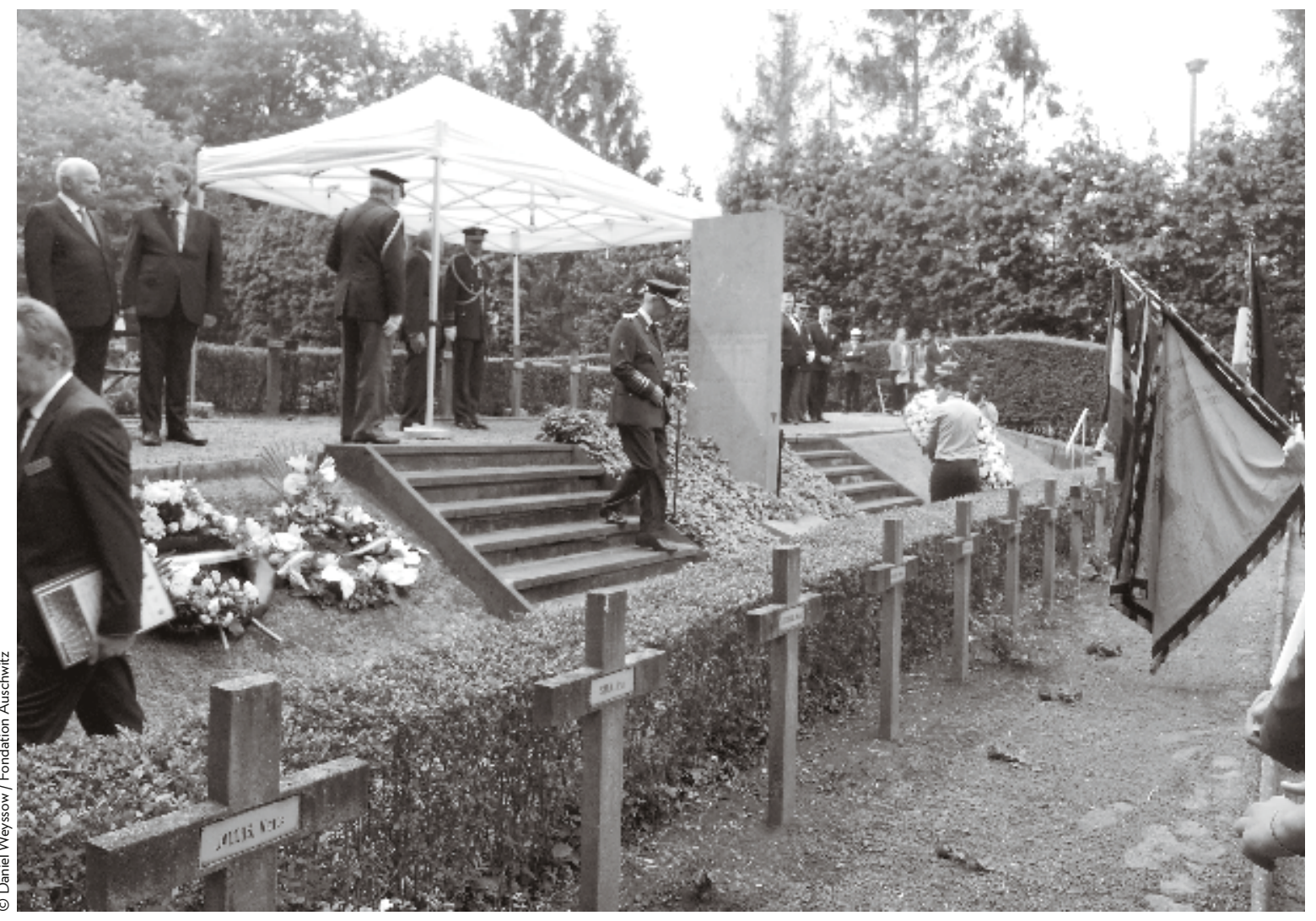


• • tion programmée. Une « cité des médias ${ }^{11}$ » surgira ici de terre d'ici 2030. Si l'Enclos des fusillés ne semble, d'après les plans disponibles, pour l'heure pas menacé, il convient de rester vigilant. Les 261 tombes de fusillés de la Seconde Guerre mondiale pourraient faire l'objet, par souci d'homogénéité autour de la vocation du futur projet, d'un compromis qui pourrait consister à déplacer les dépouilles vers d'autres pelouses. Comme cela s'était déroulé dans cette même commune de WoluweSaint-Lambert avec la transformation du cimetière dit d'Etterbeek en «Parc Georges Henri ${ }^{12}$ » il y a une vingtaine d'années.

Autre moyen, plus souple, de rencontrer et de conserver la mémoire des hommes et des femmes ayant pris part à la Seconde Guerre mondiale : les plaques mémorielles. Peu nombreuses, on les trouve apposées ci et là, au détour d'une maison, à l'exemple de celle apposée en rappel du sauvetage de jeunes filles juives cachées dans un couvent au 70 de l'avenue Clémenceau ${ }^{13}$ à Anderlecht. Le siège de la redoutable Geheime Felpolizei ${ }^{14}$ (GFP) évoquée précédemment, situé au 6 rue Traversière à Saint-Josse, n’a pas non plus été aménagé en Musée, mais en Auberge de Jeunesse. Pour ne pas transformer le rayonnement de l'artiste maudit Van Gogh (qui compta en ces lieux un ami, élève comme lui de l'Académie des Beaux-Arts) en véritable complainte de torturés, le propriétaire, qui a néanmoins accepté qu’une plaque soit posée en souvenir des résistants malmenés à cette adresse, a préféré qu'elle prenne place dans le patio intérieur plutôt qu'en façade. Elle n'est donc pas visible de l'extérieur. Son avenir étant placé sous le contrôle, aujourd'hui bienveillant mais exclusif du propriétaire, les associations mémorielles devront veiller, après l'avoir proposée, à la maintenir pour garder vivant en ces lieux le souvenir des victimes.

Et puis, il y a les plaques de rue et de square ${ }^{15}$. La dernière en date, découverte le 6 octobre 2015 à Berchem-Sainte-Agathe, a été créée en mémoire du Baron Paul Halter, dont le nom a été attribuéà un square situé à l'intersection des boulevards Mettewie et Machtens, à Molenbeek. On lui doit entre autres bienfaits comme nous l'avons vu plus haut, outre de nombreux faits de résistances durant la Seconde Guerre mondiale, la création, en 1980, de la Fondation Auschwitz, qu'il présidajusqu’à son décès survenu en 2013. Ces plaques concourent à maintenir la mémoire de personnalités qui se sont tout particulièrement distinguées, et par conséquent à maintenir éveillé le sens civique des passants.

Enfin, les pavés de mémoire ${ }^{16}$, créés par l'artiste allemand Günther Demnig, rendent hommage aux victimes de l'occupant. Chaque pavé mentionne le nom, le lieu et la date de naissance, ainsi que la date du décès - à l'exception des rares survivants - d'une personne ayant été raflée ou arrêtée. Ils sont insérés dans le trottoir, devant la porte d'entrée des derniers domiciles connus des victimes. Plus de 30000 pavés ont été disposés dans plusieurs pays européens, en majorité en Allemagne et en Autriche. Combien de temps resteront-ils en place? Tant qu'ils seront admis, souhaités, compris. C'est-àdire qu'ils seront maintenus, et remplacés s'ils devaient être abîmés, tant qu'ils conserveront une importance pour la population.

Des exemples cités, nous pouvons affirmer que sans une présence citoyenne relayée et soutenue par l'autorité publique, la création ou la sauvegarde de lieux de mémoire ne serait pas envisageable. Le maintien mémoriel des faits doublé d'une expertise demeure indispensable pour que «vivent» lieux, monuments, plaques et pavés. L'éducation à l'histoire et à la citoyenneté passe, on le sait, par un travail pédagogique à mener sans relâche notamment en milieu scolaire.

Daniel Weyssow

Mémoire d'Auschwitz ASBL

(11) http://www.editionsetseminaires.be/Presention_orateurs/ exposes_25092013/1_Emmanuel_Robben_RTBF.pdf

(12) http://arbres-inventaire.irisnet.be/medias/sites/SAM_2635.JPG

(13) http://marolles-memories.net/fr/paul-halter-clemenceau/

(14) http://www.auschwitz.be/en-nl/images/_colloques/article_gfp.jpg

(15) http://www.auschwitz.be/index.php/accueil/agenda/3044inauguration-du-square-paul-halter-6-octobre-2015

(16) http://www.auschwitz.be/en-nl/images/livschitz_3.JPG 